\title{
Gender Differences in Characteristics of Colorectal Cancer Patients: Eight Years' Experience in Tertiary Care Center.
}

\author{
Futun Aljoufi ${ }^{1}$, Adel Yasky ${ }^{1}$, Sara Qubaiban ${ }^{1}$, Roaa Amer ${ }^{1}$, Abdullah Alasaad ${ }^{1}$, Husam \\ Altahan ${ }^{1}$, Thekra AlGholaiqa ${ }^{1}$, Hala AlMarzoug ${ }^{1}$, Emad Masuadi ${ }^{1}$ Ashwaq Al Olayan ${ }^{2}$ \\ ${ }^{1}$ King Saud bin AbdulAziz University for Health Sciences, ${ }^{2}$ King AbdulAziz Medical City Riyadh, \\ KSA
}

Corresponding author: Futun Abdulrahman M. Aljoufi, Email: Futunaljoufi@gmail.com

\begin{abstract}
Introduction and Objectives: Colorectal cancer (CRC) has become a very common researched topic in Saudi Arabia. Significance of the disease lay as the third most common cancer in the Kingdom plus the various risk factors it may be connected to. Smoking, obesity, age, and gender should be further investigated in our community to focus our screening and minimize the burden of this disease. We tried to determine the differences between males and females in term of demographic, clinical and treatment characteristics. Methodology: A retrospective cohort study was performed in all cases of CRC from 2006-2014 in King Abdulaziz Medical City in Riyadh, Kingdom of Saudi Arabia (KSA). Categorical data were presented as frequencies and percentages to summarize patients' demographic data. Chi-square was used to compare between sex and the site of metastasis. Moreover, Cox regression was used to assess the risk factors on mortality. Results: Out of 581 patients diagnosed with colorectal cancer, $326(56.51 \%)$ were males. The mean age of colorectal patients at the time of diagnosis was 59.7. Majority of the BMI was overweight 183 (37.0\%) smoking history was found in only $18(3.5 \%)$ patients. Most of the patients were stage IV. The bio marker (K-RAS) was done for 189 colorectal cancer patients, of which male and female patients had mostly wild type. Tumor marker Carcinoembryonic Antigen (CEA) showed that most of the patients were in the high range for male patients $140(48.8 \%)$ and for females 115(53.2\%). Conclusion: No difference was found in regards to K-RAS mutation distribution by gender. High CEA was not associated to any age or gender. There was no statistical significance in relation to gender and response to treatment.
\end{abstract}

Keywords: Colorectal, cancer, gender, characteristics, K-RAS, CEA, cancer treatment.

\section{KEY MESSAGE}

We evaluate the differences in clinical characteristics and survival outcomes of patients with colorectal cancer who were treated at a single institution in Saudi Arabia between 2006 and 2014.

\section{INTRODUCTION}

Cancer is a term that refers to the pathology in which abnormal cells grow and divide uncontrollably, and due to their abnormal structure, they can invade nearby tissues and organs ${ }^{1}$. Colorectal cancer (CRC; pertaining to the colon and rectum) has become a common research topic in Saudi Arabia. It has gained attention due to its significance as the third most common cancer and the fourth most frequent cancer-related cause of death worldwide $^{2,3}$. However, since 2010, CRC has represented the most common type of cancer in men and the third most common type of cancer in women in Saudi Arabia ${ }^{4}$. CRC has also been a hot topic for research due to its numerous risk factors, which are usually divided into modifiable (e.g., smoking, obesity, and dietary habits) and nonmodifiable (e.g., genetics, age, and sex) factors ${ }^{3,5}$. Therefore, many studies have begun to evaluate the differences in risk factors for CRC and their impact on the incidence and mortality rates.

Another consideration is the screening process for CRC, with statistics having shown reductions in the incidence and mortality rates in the United States over the last 15 years, which has developed a well-structured screening program 6 . Nevertheless, such programs have not yet been established in Saudi Arabia, which could play a role in the 
expected dramatic increase in CRC cases. Thus, understanding the progression pattern of $\mathrm{CRC}$, along with the demographic risk factors in Saudi Arabia, could have a major influence on the decision of who to screen.

Previous studies in the United States did not demonstrate any disparities in the incidence or mortality rates of CRC between the sexes. However, internationally this consensus has changed over time ${ }^{5}$. Differences have been observed between male and female CRC patients in Saudi Arabia ${ }^{7}$. Additionally, statistics have revealed a young age of diagnosis for both sexes. In Saudi Arabia, the median age at presentation is approximately 60 years for men and slightly younger, at approximately 55 years, for women ${ }^{7}$. Thus, women are generally diagnosed at an earlier age than men.

Differences are also observed in the location of the primary tumor, with men more likely to present with rectal, rectosigmoid, and sigmoid cancers than women ${ }^{6}$. Moreover, several studies suggest that there are sex differences in the prognosis and survival of CRC patients ${ }^{3,5,7}$. The aim of this study was to evaluate the potential associations between demographic data and the pathological basis and risk factors of CRC.

\section{Patients and methods}

We conducted a retrospective cohort study of 581 patients with CRC between 2006 and 2014. The inclusion criteria were as follows: patients of both sexes aged $\geq 18$ years and a diagnosis of CRC in which colon or rectal cancer was the primary disease. All stages of CRC (Stage I-IV) were included. Patients with CRC who had other types of cancer as the primary disease were excluded, except in cases where metastases had arisen from the primary colon or rectal cancer.

The data were extracted from patient charts and our hospital information system (QuadraMed). The demographic characteristics included age at diagnosis, sex, marital status, nationality, smoking status, body mass index, a family history of cancer, and the Eastern Cooperative Oncology Group performance status at diagnosis. The clinical characteristics included the sites of metastasis,
$K-R A S$ mutation status, and Carcinoembryonic Antigen (CEA) levels. The treatment characteristics included the type of treatment and response to treatment, either by measuring CEA levels or follow-up by radiological imaging. In addition, the patient's status and cause of death were recorded.

The follow-up period was defined as the time interval between the date of diagnosis and the date of death from the disease. Deaths were confirmed by death certificates, which were available in the patients' records.

\section{Statistical analysis}

Categorical (demographic) data were presented as frequencies and percentages and continuous data (e.g., age and survival data) were presented as means, medians, and standard deviations. The Chi-square test was used to compare the categorical variables, including sex and the sites of metastasis. A Cox regression model was used to analyze risk factors for mortality. The median overall survival rate was calculated for both sexes with 95\% confidence intervals. All statistical analysis were conducted using Statistical Package for the Social Sciences for Windows, software version 20 (IMB Corp., Armonk, NY, USA). A $P<0.05$ was considered statistically significant.

\section{RESULTS}

Of the 581 patients diagnosed with CRC, 326 (56\%) were men and 255 (44\%) were women. The mean age at diagnosis was 60 (range, 2198) years. Thirty-two patients $(6 \%)$ were single, 441 patients $(88 \%)$ were married, and 32 patients $(6 \%)$ were widowed or divorced. Five hundred and sixteen patients $(89 \%)$ were Saudi; the remaining 65 patients $(11 \%)$ were non-Saudi. According to the body mass index, the majority of patients were overweight $(N=$ $183 ; 37 \%)$ or obese $(N=151 ; 31 \%)$. Twentysix patients $(5 \%)$ had a history of smoking (smokers $[N=18 ; 3 \%]$ and ex-smokers $[N=8$; $2 \%]), 215$ patients $(42 \%)$ had never smoked, and for the remaining 271 patients (53\%), the smoking history was unknown. The majority of patients $(N=426 ; 96 \%)$ did not have a family history of the disease, whereas 18 patients (4\%) did have a family history of CRC. The Eastern Cooperative Oncology 
Group performance status was 0 or 1 in the majority of patients $(N=197[51 \%]$ and $N=$ 83 [22\%], respectively).

One hundred and thirty-four male patients $(49 \%)$ and 134 female patients $(55 \%)$ had Stage IV CRC. $K$-RAS mutation analysis was performed in 334 patients. Among the male patients, $156(83 \%)$ had wild-type $K$-RAS and $33(17 \%)$ had mutated K-RAS. Similarly, among female patients, 114 (79\%) had wildtype $K-R A S$ and $31(21 \%)$ had mutated $K-R A S$. Tumor marker CEA levels were elevated in 140 male patients (49\%) and 115 female patients $(53 \%)$, respectively.

With respect to the sites of metastasis in patients with Stage IV CRC, lung, bone, liver, peritoneal, and brain metastases did not differ significantly between or in relation to the sex groups $(P>0.05)$. Lung metastasis occurred in 68 male patients $(51 \%)$ versus 52 female patients $(45 \%)(P=0.220)$. Bone metastasis occurred in 16 male patients (12\%) versus 15 female patients $(13 \%)(P=0.950)$. Liver metastasis occurred in 102 male patients (76\%) versus 90 female patients $(78 \%)(P=$ $0.703)$. Peritoneal metastasis occurred in 21 male patients (16\%) versus 11 female patients $(10 \%)(P=0.111)$.

Regarding the response to treatment in the different sex groups, no statistically significant association between sex and treatment response was observed $(P=0.525)$. Sixty-four male patients $(62 \%)$ and 60 female patients (66\%) were non-responders and 40 male patients (38\%) and 31 female patients (34\%) were responders.

In response to radiological treatment, 9 male patients $(8 \%)$ exhibited a partial response, 4 (4\%) exhibited a complete response, 35 (30\%) had stable disease, and $67 \quad(58 \%)$ had progressive disease. Among the female patients, 4 (4\%) exhibited a partial response, 6 (7\%) exhibited a complete response, 28 (31\%) had stable disease, and 52 (58\%) had progressive disease. One hundred and thirtyfour male patients (54\%) and 115 female patients $(46 \%)$ responded to surgical treatment and 134 male patients (54\%) and 115 female patients $(46 \%)$ responded to chemotherapy.
Fifty-nine male patients (44\%) and 47 female patients $(41 \%)$ died following treatment. Sixty male patients $(45 \%)$ and 51 female patients (44\%) were alive and 32 patients $(26 \%)$ were lost to follow-up. Cancer-related deaths occurred in 51 male patients $(90 \%)$ and 38 female patients (93\%), while non-cancerrelated deaths occurred in 6 male patients $(10 \%)$ and 3 female patients (7\%).

The survival analysis is summarized in figure 1 and 2. No significant differences in survival were observed between the sexes $(P=0.976)$. The median overall survival times for male and female patients were 54.3 months and 41.8 months, respectively. Age, $K-R A S$ mutation status, and the number of metastases were not significant risk factors for CRC in these patients $(P>0.05)$.

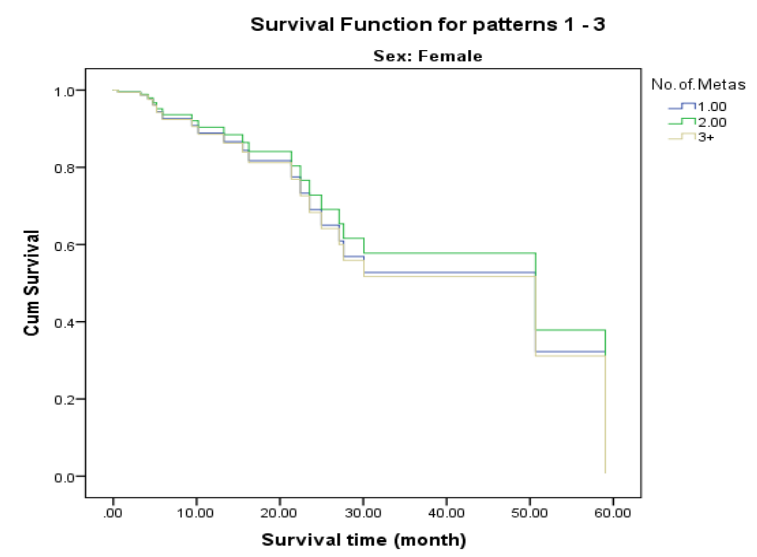

Fig 1: Survival analysis, female.

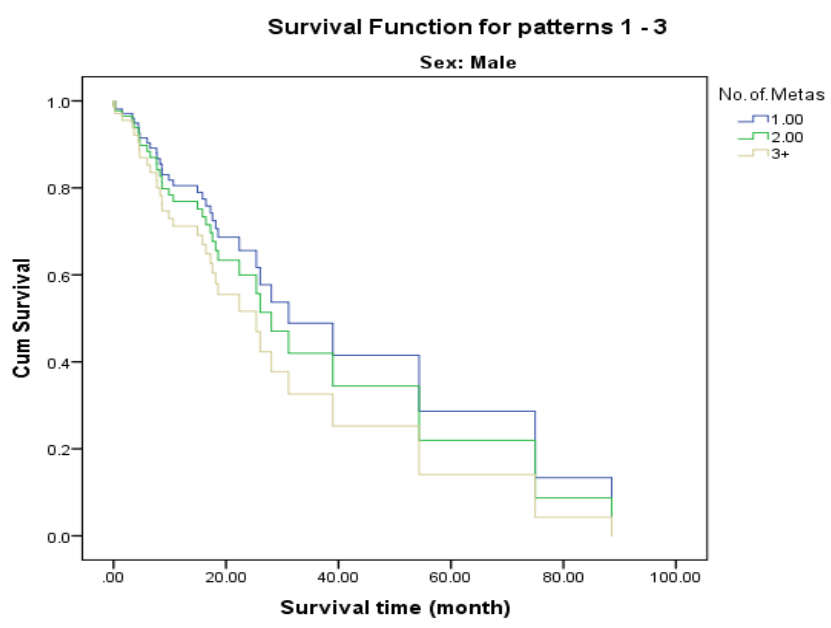

Fig 2: Survival analysis, male. 


\section{DISCUSSION}

Worldwide prevalence points to CRC being the third most common cancer in men and the fourth most common cancer in women ${ }^{6}$. In Saudi Arabia, CRC has been ranked as the most common cancer in men and the third most common cancer in women, according to the Saudi Cancer Registry ${ }^{4}$. A study conducted in China demonstrated that $K-R A S$ mutations were more prevalent in women than in men $^{8}$. However, a study conducted in Spain has revealed no significant differences in respect of the distribution or frequency of $K-R A S$ mutations according to sex ${ }^{9}$. Similarly, a study conducted in Saudi Arabia in 2014 concluded that there was no significant association between the frequency of $K-R A S$ mutations and $\operatorname{sex}^{10}$. We detected $K$-RAS mutations in $17.5 \%$ of male patients and $21.4 \%$ of female patients, respectively. Our results also showed that elevated serum tumor marker CEA levels were detected in $48.8 \%$ of male patients and $53.2 \%$ of female patients, respectively. However, a study published in 2015 suggested that tumor marker CEA levels were not associated with various factors, including age and $\operatorname{sex}^{11}$. Women exhibited a tendency towards developing a greater number of metastases $(\geq 3: 56 \% v s$. $44 \%$ for female $v s$. male). Furthermore, women also exhibited a slightly lower tendency towards developing bone metastases than men, which is comparable to the findings of a previous study conducted in India ${ }^{12}$. Additionally, lung, liver, and peritoneal metastases were detected more frequently in men than in women. However, for other previously mentioned types of metastasis, the frequency was higher in women than in men, which was statistically significant.

Our findings revealed no statistical significance in relation to sex or response to treatment. Sex-specific barriers play a role in the optimal management of CRC, and this is a rich area for further research ${ }^{13}$. In the present study, we found that the mortality rate was higher in male patients $(56 \%)$ than in female patients (44\%). Similarly, a study conducted in Germany reported that the age-adjusted 5-year relative survival rate was higher in female patients than in male patients $(65 \%$ vs. $62 \%$, respectively; $P<0.001)^{14}$. However, another study showed that female patients aged $>55$ years tended to have a significantly poorer survival than male patients ${ }^{15}$.

\section{CONCLUSION}

Our study showed a higher mortality rate in males with no significant difference in $K-R A S$ mutation distribution between genders. High CEA did not relate to any specific age or gender. Furthermore, there was no response variation between genders in regard to treatment. Efforts should be made to further investigate differences and similarities between genders when it comes to colorectal cancer especially in the aspect of treatment.

\section{REFERENCES}

1. National Cancer Institute (2017): What is Cancer? [Online] Available from: https://www.cancer.gov/aboutcancer/understanding/what-is-cancer.

2. World Cancer Research Fund and American Institute for Cancer Research. (2007): Food, Nutrition, Physical Activity and the Prevention of Cancer: A Global Perspective. Washington, DC: American Institute for Cancer Research. From: https://www.ncbi.nlm.nih.gov/pubmed /10378216

3. Haggar FA, Boushey RP (2009): Colorectal cancer epidemiology: incidence, mortality, survival, and risk factors. Clin Colon Rectal Surg., 22(4): 191-197.

4. Saudi Cancer Registry (2014): Cancer Incidence Report. Riyadh: Council of Health Services, Kingdom of Saudi Arabia. Available from: http://www.chs.gov.sa/Ar/mediacenter /NewsLetter/2010\%20Report\%20(1).p df.

5. Matanoski G, Tao X, Almon L et al. (2006): Demographics and tumor characteristics of colorectal cancers in the United States, 1998-2001. Cancer, 107(5): 1112-1120.

6. Sibiani AR, Fallatah HI, Akbar HO et al. (2011): Colorectal Cancer in Saudi Arabia King Abdul Aziz University Hospital: A Five Year Experience. J Med Med Sci., 2(10): 1126-1130. 
7. Alsanea NA (2015): The acceptability of screening for colorectal cancer in Saudi Arabia: myths busted. Saudi J Gastroenterol., 21(2): 59.

8. Li W, Qiu T, Ling Y et al. (2015): Molecular pathological epidemiology of colorectal cancer in Chinese patients with KRAS and BRAF mutations. Oncotarget., 6(37): 3960739613.

9. Roa I, Sánchez T, Majlis A, Schalper K (2013): [KRAS gene mutation in colorectal cancer]. Rev Med Chil., 141(9): 1166-1172.

10. Zahrani A, Kandil M, Badar T et al. (2014): Clinico-pathological study of $\mathrm{K}$-ras mutations in colorectal tumors in Saudi Arabia. Tumori., 100(1): 7579.

11. Zhong W, Yu Z, Zhan J et al. (2015): Association of serum levels of CEA, CA199, CA125, CYFRA21-1 and CA72-4 and disease characteristics in colorectal cancer. Pathol Oncol Res., 21(1): 83-95.

12. Suresh Babu MC, Garg S, Lakshmaiah KC et al. (2017): Colorectal cancer presenting as bone metastasis. J Cancer Res Ther., 13(1): 80-83.

13. Kim SE, Paik HY, Yoon $\mathrm{H}$ et al. (2015): Sex- and gender-specific disparities in colorectal cancer risk. World J Gastroenterol., 21(17): 51675175.

14. Majek O, Gondos A, Jansen L et al. (2013): Sex differences in colorectal cancer survival: population-based analysis of 164,996 colorectal cancer patients in Germany. PLoS ONE, 8(7): e68077.

15. Hendifar A, Yang D, Lenz F et al. (2009): Gender disparities in metastatic colorectal cancer survival. Clin Cancer Res., 15(20): 6391-6397. 